\title{
Influencia de la apatía en adolescentes de primer grado en secundaria pública sobre planeación y toma de decisiones
}

\author{
Influence of apathy in first grade adolescents in public high school on planning and decision \\ making
}

\author{
María de la Paz Osuna-Fernández ${ }^{a}$, Daniel Álvarez-Núñez ${ }^{b}$
}

\begin{abstract}
:
The present study analyzed the influence of apathy in planning and decision making in adolescents. With a population of 249 firstgrade teenage students in the Public High School, EEVA apathy scale was registered to obtain a sample of 84 participants, three groups of 28 participants each were formed: Control group (GC) with low apathy, two with high apathy experimental group (GE) and experimental group with reward (GER). Subsequently, the planning task will be applied with the Tower of Hanoi test and the decision making with the Card Game. The results show that in the Hanoi test there are differences in the number of movements in the control and experimental groups with reward.
\end{abstract}

Keywords:

Apathy, executive functions, planning, decision making, adolescence, motivation

Resumen:

El presente estudio analizó la influencia de la apatía en la planeación y toma de decisiones en adolescentes. Con una población de 249 adolescentes estudiantes de primer grado en Escuela Secundaria Pública, se utilizó escala de apatía EEVA para la obtención de una muestra de 84 participante, se conformaron tres grupos de 28 participantes cada uno: Grupo control (GC) con apatía baja, dos con apatía alta grupo experimental (GE) y grupo experimental con recompensa (GER). Posteriormente se aplicaron tarea de planeación con prueba Torre de Hanoi y toma de decisión con Juego de Cartas. Los resultados muestran que en la prueba de Hanoi hay diferencias en el número de movimientos en los grupos control y experimental con recompensa.

Palabras Clave:

Apatía, funciones ejecutivas, planeación, toma de decisión, adolescencia, motivación

\section{Introducción}

En las últimas décadas se ha puesto de manifiesto la importancia de considerar la apatía como un síndrome que tiene un impacto social, edu cativo y de salud pública relevante en la sociedad [1].

La apatía se define como un síndrome que se manifiesta por la reducción en la motivación, pensamientos, emociones y conducta dirigido a objetivos, la cual no es atribuible a disminución de la conciencia, déficit intelectual o alteraciones afectivas. [2].
En la investigación clínica, se pone de manifiesto la apatía asociada a alteraciones neurológicas como son demencia, enfermedad de Alzheimer y lesiones frontales, la cual ocurre cuando los sistemas que generan y controlan la acción voluntaria están alterados. Estos sistemas están en su mayoría representados por diferentes regiones, interconectadas de la corteza pre frontal y ganglios basales [3].

En algunos estudios se encontró que los pacientes

\footnotetext{
Autor de Correspondencia, Centro de Enseñanza Técnica y Superior, https://orcid.org/0000-0002-4444-9614 Email: madepazz@gmail.com
}

b Profesor investigador de tiempo completo de la Escuela de Psicología del Centro de Enseñanza Técnica y Superior, https://orcid.org/00000003-2883-9572 Email: daniel.alvarez@cetys.mx 
apáticos con enfermedades de Alzheimer mostraron déficits significativamente mayores en evaluaciones de funcionamiento ejecutivo. [4]. Existe evidencia de estudios neuropsicológicos que la apatía puede ser asociada con disfunción ejecutiva

El poblaciones clínicamente sanas, la apatía es selectiva, en donde un individuo carece de motivación o interés en una actividad en particular [2]. Estudios ampliaron la investigación y sugirieron 3 factores: (1) apatía ejecutiva que se refiere a funciones ejecutivas como la atención y la planeación; (2) apatía emocional que es la integración de comportamientos emocion ales; y la apatía de iniciación cognitivo y conductual [1].

Con base en lo anterior, existe evidencia que la apatía puede incidir en procesos ejecutivos, entre ellos la planeación y la toma de decisión, sin embargo no se ha estudiado esta relación en adolescentes. Cabe mencionar que, en estudios recientes se ha puesto de manifiesto que en la adolescencia ocurre un cambio significativo a medida que los estudiantes aprenden a separar la escuela y el aprendizaje, [5]. A esta distinción se le ha denominado el "Juego de la Escuela" donde la escolarización se convierte en un entorno basado en la estructura, normas y el cumplimento de objetivos. Sumando a esto, el desarrollo de estructuras frontocorticales en esa edad está en proceso. Existe evidencia que la adolescencia se caracteriza por presentar mayor sensibilidad hacia la recompensa en la toma de decisión [6].

El objetivo de este estudio es determinar el rendimiento en tareas de planeación y toma de decisiones en adolescentes que presentan apatía, así como las diferencias de puntaje en tareas de dichos procesos en adolescentes con apatía brindándose recompensa.

\section{Método}

\section{Participantes}

Participaron en el estudio un total de 249 adolescentes de los cuales se obtuvo una muestra de 84 participantes de ambos sexos con un rango de edad que oscila entre 12 a 13 años, los cuales cursan el Primer grado en la Escuela Secundaria Pública Mariano Sánchez en el Municipio de Ensenada, México. El criterio de exclusión para participar en el estudio fue presentar depresión y apatía moderada. Después de la aplicación de la Escala para evaluar la apatía EEVA, se conformaron tres grupos: Grupo control (GC) 28 participantes con apatía baja, grupo experimental (GE) 28 participantes con apatía alta y grupo experimental con recompensa (GER) 28 participantes, con un total de 84 participantes, 42 mujeres y 42 hombres. La participación de los adolescentes fue voluntaria, mediante un consentimiento informado a los padres de familia y a los adolescentes. Todos los procedimientos involucrados en ésta investigación fueron aprobados por el Comité de Ética Institucional de CETYS Universidad, de acuerdo con los estándares éticos establecidos en la Declaración de Helsinki de 1964.

\section{Instrumentos}

Para la evaluación de Apatía se utilizó la Escala para evaluar la apatía (EEVA) [2]. EEVA ha sido validada con estudiantes del Estado de México, está compuesto por preguntas cerradas y con un formato de cinco opciones de respuesta. Es un auto informe que está compuesta por un total de 51 ítems redactados en forma de afirmaciones divididos en tres componentes: 17 conductuales, 17 cognitivos y 17 afectivos en formato tipo Likert. Los puntajes totales oscilan de 51 a 255 puntos y se interpretan así: entre 51 y 69 indican apatía baja; entre 70 y 112 , se considera apatía moderada y los puntajes totales entre 113 y 204 puntos, indican una alta apatía [2].

Inventario de Beck ((BDI por sus siglas en inglés) [Beck \&Steer, 1984; Mukhtar \&Tian, 2008]. El Inventario de Depresión de Beck, IA (BDI-IA), estandarizado por Jurado et al., (1998) en población mexicana, es un auto informe de 21 ítems, referidos a síntomas depresivos en la semana previa a la aplicación, con cuatro opciones de respuesta. Los puntajes de severidad de síntomas van de 0 a 63, donde 63 representa la severidad máxima [7].

Dentro de las pruebas que se utilizan para evaluar la capacidad de planificación se encuentra la Torre de Hanoi. El objetivo de ésta prueba es evaluar la capacidad de planeación secuencial, la cual requiere de realizar diversos pasos intermedios en una secuencia (que en ocasiones contiene pasos contra-intuitivos: aparentemente aleja del objetivo planteado porque va en sentido inverso) para llegar a una meta final. A los participantes se les presentó la Torre de Hanoi la cual es figura de madera con tres postes verticales de la misma longitud fijados en una base horizontal. En el poste de la izquierda estaban colocados cuatro cilindros de madera de tamaños progresivamente mayores ordenados de manera que el inferior era el más grande y el superior el más pequeño. Se les dio la instrucción que debían de considerar 3 reglas las cuales eran mover los cilindros de uno en uno y de poste en poste hasta situarlos en la misma posición inicial, pero en el poste más a la derecha, segundo un cilindro no podía colocarse sobre uno de tamaño menor y finalmente no podía colocar ningún cilindro fuera de la estructura de madera. [8] 
En la aplicación de la tarea se registra el número de movimientos que el participante realizó para llegar a la meta final (la colocación de los cilindros en el poste correspondiente), y el tiempo en segundos que utilizó en la tarea.

Para la evaluación de toma de decisión se utilizó la prueba de Juego de Cartas versión incluida en la Batería neuropsicológica BANFE-2. Esta prueba consiste en ir eligiendo cada carta conforme a un criterio propio, teniendo en cuenta los riesgos y beneficios de la elección, con el objetivo de lograr la mayor cantidad de puntos está integrado por cinco mazos, cada uno con 18 cartas, cuyos valores van de 1 a 5 puntos. En la tarea de toma de decisión el individuo no tiene información previa acerca de las características de los mazos antes de empezar a jugar. En la consigna no se le dice al participante la existencia de mazos buenos y malos. Esto tiene la finalidad de hacer que el aprendizaje de la toma de decisiones no tenga ningún sesgo acerca de lo que debería preferir la persona. Tampoco el participan te ve la suma de los puntos que lleva ganado o perdido, sólo se le informa cuántos puntos gana o pierde cada vez que levanta una carta. Este último procedimiento, impide que el participante tenga una contabilidad exacta de lo que gana y pierde y deba guiarse aún más por las impresiones que se va formando. [9]

En la prueba de Juego de Cartas se cuenta con mazos de riesgo y mazos de beneficio, donde el participante va a detectar y seleccionar las cartas de su elección. Durante la tarea se registró el porcentaje de cartas de riesgo que eligió el participante.

\section{Procedimiento}

Los participantes fueron evaluados en la mañana durante los meses de Octubre a noviembre de 2019, en una sesión de 30 minutos cada una, en las instalaciones de la misma secundaria donde los estudiantes están cursando sus asignaturas, los alumnos fueron requeridos directamente de su aula y llevados a una habitación libre de ruidos y estímulos distractores, especialmente acondicionados para este fin. En el grupo experimental la recompensa consistió en proporcionar un punto en la asignatura de matemáticas, la cual es una materia donde se reflejan altos índices de reprobación escolar.

\section{Análisis estadístico}

Para determinar si hubo diferencias entre los grupo se realizó un ANOVA una vía para muestras independientes para evaluar la relación entre la variable Apatía y el desempeño en Torre de Hanoi con respecto al número de movimientos. En las pruebas de Juego de cartas y Tiempo estimado de ejecución (torre de Hanoi, en relación con apatía, se realizó un análisis estadístico mediante Kruskal-Wallis, debido a que los datos no mostraron una distribución normal.

\section{Resultados}

\section{Torre de Hanoi}

El análisis del número de movimientos mostró que en grupo control obtuvo una media de 33.17 movimientos (desv. 14.40), para los participantes del grupo experimental se obtuvo una media de 36.75 (DS=14.26), y el grupo de participantes con recompensa obtuvo un 44.69 (desv. 16.52). El análisis estadístico realizado reveló diferencias significativas $[F(2,83)=4.414, p<0.05]$, entre los grupos. El análisis de comparaciones múltiples confirmó que el grupo que tuvo un mayor promedio en la prueba de torre de Hanoi fue el grupo experimental con recompensa.

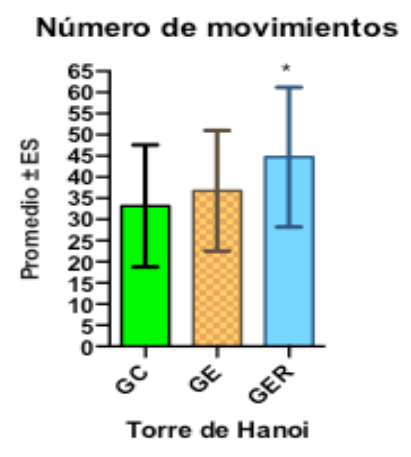

Figura 1.Análisis ANOVA muestra número de movimientos utilizados y los tres grupos de estudio con apatía.

Los resultados del análisis para evaluar la relación del tiempo utilizado durante la ejecución de la tarea de Torre de Hanoi en los tres grupos de estudio. Mediante el análisis estadístico manifiesta que no hay diferencias significativas $p=0.1105$, en los participantes de grupo control y experimental con y sin recompensa. Ver figura 2

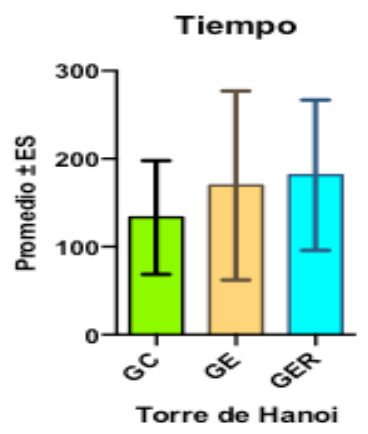


Figura 2.Análisis Kruskal-Wallis muestra el tiempo utilizado durante la tarea de Torre de Hanoi en los tres grupos de estudio.

\section{Juego de Cartas}

En tarea de toma de decisión con prueba de Juego de Cartas se realizó un análisis mediante Kruskal-Wallis, para determinar el porcentaje de cartas de riesgo en los participantes con apatía baja y alta, así como los participantes con recompensa. La figura 3 nos muestra que no hay diferencias significativas $p=0.1294$ en la comparación de los 3 grupos de estudio.

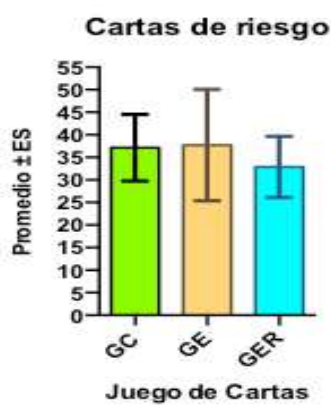

Figura 3.Análisis Kruskal-Wallis muestra la relación en el porcentaje de cartas de riesgo entre los tres grupos de estudio.

\section{Discusión}

El objetivo del estudio es determinar el rendimiento en planeación y toma de decisiones en adolescentes que presentan apatía, así como las diferencias de puntaje en tareas de dichos procesos en adolescentes con apatía brindándose recompensa.

Se encontraron diferencias estadísticamente significativas en total de los movimientos ejecutados en prueba de Hanoi por los adolescentes entre los grupos control y el experimental con recompensa (ver figura 1), siendo el grupo con recompensa el que obtuvo mayor puntaje, esto puede ser explicado, con estudios que revelan que los adolescentes tienden a tomar decisiones que los benefician a corto plazo, Los cambios hormonales durante este periodo de la adolescencia dan como resultado que el núcleo accumbens, la parte del cerebro que proporciona placer cuando se recompen sa, tenga mayor sensibilidad, como resultado son más propensos a ser más impulsivos para satisfacer este placer de recompensa. $10 \mathrm{Sn}$ embargo, en las tareas de toma de decisión y tiempo de ejecución no se muestran diferencias significativas que nos indiquen que la apatía está relacionada con el rendimiento en planeación y toma de decisiones.

Cabe mencionar que los resultados reflejan inconsistencia, con base a que el tiempo de ejecución del grupo experimental con recompensa obtuvo una tendencia (figura2) mayor en el tiempo requerido para la ejecución de Torre de Hanoi. Estos resultados coinciden con estudios realizados con adolescentes en los cuales se muestran resultados inciertos, esto considerando la etapa de la adolescencia está caracterizada por el desarrollo de estructuras frontocorticales necesarias para el trabajo de procesos ejecutivos. [11]

Una de las limitaciones que subyacen al estudio de la apatía en relación con otros procesos cognitivos es la falta de instrumentos validados y estandarizados en México, que no solo sean en forma de auto informe, sino también valoraciones de informante, reconociendo la necesidad de una identificación confiable de la apatía y buscando formas para una buena comunicación tanto en la investigación como en el tratamiento.

\section{Referencias}

[1] Ross S.M., Dootson P. (2018) Do I Care? Pathological Apathy in the Context of Sustainable Consumption. In: Krey N., Rossi P. (eds) Back to the Future: Using Marketing Basics to Provide Customer Value. AMSAC 2017. Developments in Marketing Science: Proceedings of the Academy of Marketing Science. Springer, Cham

[2] Marin, R. (1990). Differential diagnosis and classification of apathy. Am J Psychiatry, 147(1), 22-30.

[3] Levy, R., \& Dubois, B. (2005). Apathy and the Functional Anatomy of the Prefrontal Cortex-Basal Ganglia Circuits. Cerebral Cortex, 16(7), 916-928.

[4] Frakey L.L. (2018) Apathy. In: Kreutzer J.S., DeLuca J., Caplan B. (eds) Encyclopedia of Clinical Neuropsychology. Springer, Cham

[5] Marshall J.C. (2012) Apathy in Learning. In: Seel N.M. (eds) Encyclopedia of the Sciences of Learning. Springer, Boston, MA

[6] Galván, A., \& McGlennen, K. M. (2013). Enhanced Striatal Sensitivity to Aversive Reinforcement in Adolescents versus Adults. Journal of Cognitive Neuroscience, 25(2), 284-296.

[7] Beltrán, María del Carmen, Freyre, Miguel-Ángel, \& HernándezGuzmán, Laura. (2012). El Inventario de Depresión de Beck: Su validez en población adolescente. Terapia psicológica,30(1),5-13. 
[8] Díaz, A., Martín, R., Jiménez, J. E., García, E., Hernández, S., \& Rodríguez, C. (2012). Torre de Hanoi: datos normativos y desarrollo evolutivo de la planificación. European Journal of Education and Psychology, 5(1), 79-91.

[9] Squillace Louhau, M. R., Picón Janeiro, J. C., \& Schmidt, V. I. (2015) Juego de Cartas, una tarea para la evaluación de toma de decisiones riesgosas: aportes a la validez ecológica y de constructo.

[10] Widjojo, K. J Youth Adolescence (2018) 47: 1789. https://ebiblio.cetys.mx:4083/10.1007/s10964-018-0890-x

[11] Boyer T.W., Byrnes J.P. (2016) Risk-Taking. In: Levesque R. (eds) Encyclopedia of Adolescence. Springer, Cham 